\title{
Polymorphisms of rs4787050 and rs8045980 are associated with lung cancer risk in northeast Chinese female nonsmokers
}

\author{
Xiaoying $\mathrm{Li}^{1,2}$, Xuelian $\mathrm{Li}^{2}$, Zhihua Yin'², Min Jiang ${ }^{1,2}$, Wen Tian ${ }^{1,2}$, Man Tang ${ }^{3}$ \& Baosen \\ Zhou*,1,2 \\ ${ }^{1}$ Department of Clinical Epidemiology, First Affiliated Hospital of China Medical University, Shenyang 110001, PR China \\ ${ }^{2}$ Department of Epidemiology, School of Public Health, China Medical University, Shenyang, 110122, PR China \\ ${ }^{3}$ Department of Clinical Pharmacology, School of Pharmacy, China Medical University, Shenyang, 110122, PR China \\ *Author for correspondence: Tel.: +86 1338688 1563; bszhou@cmu.edu.cn
}

\begin{abstract}
Aim: We studied the association between two single-nucleotide polymorphisms (SNPs: rs4787050 and rs8045980) in RBFOX1 and lung cancer risk, and explored the interaction between the two SNPs and exposure to cooking oil fume on lung cancer risk in northeast Chinese female nonsmokers. Methods: Northeast Chinese female nonsmokers were enrolled into the study (people with lung cancer, 647; people without lung cancer, 675). All statistical analyses were performed using SPSS software. Results: The SNPs rs 4787050 and $r 8045980$ showed a significant association with susceptibility to lung cancer. Moreover, cooking oil fume exposure was found to increase the risk of lung cancer. However, no gene-environment interactions were discovered. Conclusion: The present study revealed that rs4787050 and rs8045980 in RBFOX1 may be meaningful as a novel biomarker for lung cancer susceptibility.
\end{abstract}

First draft submitted: 19 December 2018; Accepted for publication: 15 May 2019; Published online: 12 September 2019

Keywords: female nonsmokers $\bullet$ lung cancer $\bullet$ RBFOX1 $\bullet$ single-nucleotide polymorphisms $\bullet$ susceptibility

Lung cancer is one of the most frequently diagnosed malignancies worldwide, with a high rate of morbidity and mortality [1-3]. In China, lung cancer is the leading cause of cancer mortality among both men and women [4]. Although smoking is the leading risk factor for lung cancer [5], some lung cancer cases, especially among women, are not related to a history of smoking [6], suggesting the role of other risk factors that are likely associated with the disease risk. Molecular epidemiologic studies have revealed the involvement of many genes in causing lung cancer [7]. Thus, exploring the association between gene polymorphisms and lung cancer in female nonsmokers is of immediate concern.

RBFOX1 (or 2BP1, FOX1, A2BP1, FOX-1 and HRNBP1), located in the chromosome 16p13.3, encodes Fox-1 family proteins. RBFOX proteins are RNA-binding proteins that include three paralogs in mammals: RBFOXI, RBFOX2 and RBFOX3 [8]. Jin et al. [9] first demonstrated that Fox-1 family proteins could regulate alternative splicing via (U)GCAUG elements. Later, Klorin et al. [10] reported that RBFOX1 may be a new candidate tumor suppressor gene, warranting further research. Earlier studies have also demonstrated the association of RBFOXI with various types of cancers, such as liver, lung, gastric and breast cancer - among others. [10-18]. Furthermore, Cancer Cell Line Encyclopedia website predicts a higher expression of $R B F O X 1$ in various cancer cell lines, including lung cancer cell lines, than that in normal cell lines.

Single-nucleotide polymorphisms (SNPs) - the most common form of gene alteration in the human genome - refers to single-nucleotide variations with distribution frequencies $>1 \%$ in the population. SNPs have a great impact on tumorigenesis and tumor development [19-21]. Although the association between SNPs in RBFOX1 and cancer has been explored in some previous studies [11,14,22], the association between SNPs in RBFOXI and lung cancer risk remains unclear. Rs4787050 and rs8045980 (located at 7618998 and 7601190 on chromosome 16, respectively) are located on the intron region of RBFOX1. We chose these SNPs as they do not code for any proteins, they seem to play the role of a modifier, indicating that they may regulate and affect $R B F O X 1$ transcription 
(http://useast.ensembl.org/info/docs/tools/vep/index.html). Xue et al. revealed that exposure to cooking oil fume may increase the risk of lung cancer in Chinese female nonsmokers [23]. Le et al. found that gene-environment interaction may play a vital role in lung cancer risk [24]. Therefore, the present study intended to elucidate the association between SNPs (rs4787050 and rs8045980) in RBFOX1 and lung cancer risk, as well as analyze the interaction between SNPs and exposure to cooking oil fume on lung cancer risk among northeast Chinese female nonsmokers, which may provide a new screening strategy for lung cancer in female nonsmokers.

\section{Materials \& methods}

Study subjects

This hospital-based, case-control study was conducted in the Shenyang City, located in northeast China, involving 1322 participants (patients with lung cancer: 647; people without lung cancer: 675). The inclusion and exclusion criteria for cases were according to our previous study [25]. The inclusion criteria were as follows: newly diagnosed lung cancer without chemoradiotherapy and nonsmoking female patients. The exclusion criteria were as follows: a history of previous cancer or metastasized cancer from a different tumor. All study patients were recruited from the affiliated hospital of China Medical University between March 2010 and January 2014. Meanwhile, age-matched ( \pm 5 years) control subjects without a history of cancer were selected from medical examination centers of the same hospital. Environmental exposure was defined as described previously [26]. All subjects were genetically unrelated Han-Chinese, nonsmoking women. This research was supported by the Institutional Review Board of China Medical University, and informed consent was obtained from all participants.

\section{DNA genotyping}

At first, $5 \mathrm{ml}$ venous blood sample was drawn from every participant and was then used to isolate genomic DNA based on the phenol-chloroform method. A 7500 Fast Real-time PCR system (Applied Biosystems, CA, USA) was used to perform genotyping. PCR Taqman primers and probes (C_2596054_10 for rs4787050, C_29181896_10 for rs8045980) were designed by Applied Biosystems (CA, USA). The reaction conditions was as follows: firstly $10 \mathrm{~min}$ at $95^{\circ} \mathrm{C}$, then 47 cycles of $92^{\circ} \mathrm{C}$ for $30 \mathrm{~s}$, finally $1 \mathrm{~min}$ at $60^{\circ} \mathrm{C}$. To control quality, the researchers were unaware of the case-control statue and we randomly selected about $10 \%$ samples to duplicate analysis. Results of duplicated genotyping were totally in accordance with the former ones.

\section{Statistical analysis}

All statistical analyses were performed with SPSS software (version 20.0) and were two-sided. $\mathrm{p}<0.05$ was defined as the criterion of statistical significance. A goodness-of-fit $\chi^{2}$ test was conducted to assess Hardy-Weinberg equilibrium [27]. The $\chi^{2}$ and t-test (or the Mann-Whitney $U$ test) were used to evaluate the distribution of categorical variables and continuous variables between people with and without lung cancer, respectively. The unconditional logistic regression analysis (referring to enter method of analysis) was performed to calculate the odds ratios (ORs) and 95\% confident intervals (CIs), which could estimate association between SNPs and lung cancer risk. The linkage disequilibrium (LD) analysis was conducted using SHEsis online web-server [28]. The crossover analysis was used to evaluate gene-environment interaction. Interactions in the additive model were assessed according to Tomas Andersson's study [29]. Interactions in the multiplicative model were estimated by logistic regression model. p-values and ORs were adjusted by age.

\section{Results}

Baseline characteristics

The basic characteristics of 647 patients and 675 control subjects were summarized in Table 1. All participants were nonsmoking women, and the vast majority of the people with lung cancer studied were adenocarcinomas. No significant difference was found in terms of age $(\mathrm{p}=0.311$; this was evaluated by the Mann-Whitney $\mathrm{U}$ test), education $(\mathrm{p}=0.392)$, family history $(\mathrm{p}=0.430)$ and passive smoking $(\mathrm{p}=0.382)$ between the case and control groups, with their mean ages being $55.828 \pm 13.661$ and $56.832 \pm 11.734$ years, respectively. However, a significant difference was noted in exposure to cooking oil fume $(\mathrm{p}=0.002)$ between the groups. In the case group, 440 patients had adenocarcinoma, 104 patients had squamous cell carcinoma, 94 patients had small cell lung cancer and nine patients had other types. 


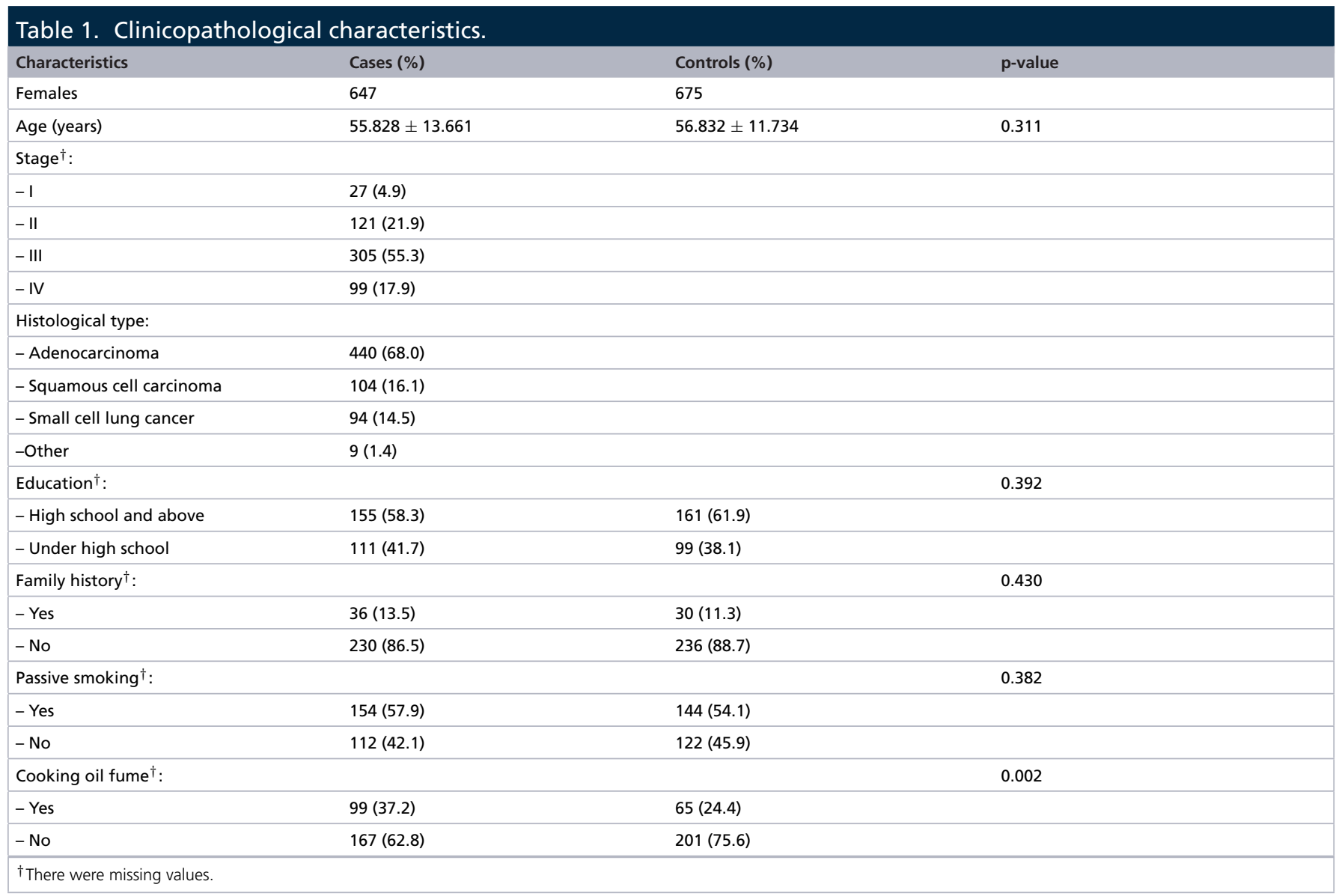

\section{Association between rs4787050/rs8045980 \& lung cancer risk}

The observed genotype frequencies of rs4787050 and rs8045980 among the control subjects conformed to the Hardy-Weinberg equilibrium $\left(\chi^{2}=1.403 ; p=0.236 ; \chi^{2}=0.906 ; p=0.341\right.$, respectively). The frequencies of SNPs and their associations with lung cancer susceptibility were summarized in Table 2. For the distribution of rs4787050 and rs8045980, statistically significant differences were observed between the groups. Even after Bonferroni correction, the two SNPs were still associated with susceptibility to lung cancer. For rs4787050, considering homozygous wild AA genotype as the reference group, GG genotype (adjusted odds ratio $[\mathrm{OR}]=2.471,95 \% \mathrm{CI}$ $=1.262-4.836 ; \mathrm{p}=0.008)$ and the dominant model $(\mathrm{AG}+\mathrm{GG}$; adjusted $\mathrm{OR}=1.323,95 \% \mathrm{CI}=1.051-1.665 ; \mathrm{p}$ $=0.017)$ were associated with a significantly increased risk of lung cancer. In the recessive model, the GG genotype of rs 4787050 revealed a significant increase in the risk of lung cancer in comparison with the reference group (AA + AG; adjusted $\mathrm{OR}=2.310,95 \% \mathrm{CI}=1.185-4.503 ; \mathrm{p}=0.014)$. Moreover, allele comparison demonstrated that the $\mathrm{G}$ allele of rs 4787050 was related to a higher risk of lung cancer than the $\mathrm{A}$ allele $(\mathrm{OR}=1.340,95 \% \mathrm{CI}=1.098$ $1.635 ; \mathrm{p}=0.004)$. For rs8045980, subjects carrying heterozygous AC or homozygous AA genotype showed an increased risk of lung cancer compared with those carrying homozygous wild CC genotype (adjusted OR $=1.448$, $95 \% \mathrm{CI}=1.150-1.825 ; \mathrm{p}=0.002$; adjusted $\mathrm{OR}=1.629,95 \% \mathrm{CI}=1.125-2.357 ; \mathrm{p}=0.010$, respectively). In the dominant model, the AC + AA genotype of rs 8045980 was associated with a significantly increased risk of lung cancer compared with the reference ( $r s 8045980-C C$; adjusted $\mathrm{OR}=1.480,95 \% \mathrm{CI}=1.186-1.846 ; \mathrm{p}=0.001$ ). Allele comparison suggested that rs $8045980-\mathrm{A}$ allele increased the susceptibility to lung cancer in comparison to the $\mathrm{C}$ allele $(\mathrm{OR}=1.303,95 \% \mathrm{CI}=1.110-1.529 ; \mathrm{p}=0.001)$.

\section{Stratification analyses \& the linkage disequilibrium analysis}

To further clarify the association between the two SNPs in RBFOX1 and lung cancer risk, we performed stratified analyses according to the histological types. The results of lung adenocarcinoma were listed in Table 3 . For rs 4787050 , subjects with the $G$ allele showed a statistically elevated risk of lung adenocarcinoma compared with 
Table 2. Relationship analyses of single-nucleotide polymorphisms in RBFOX1 with lung cancer risk.

\begin{tabular}{|c|c|c|c|c|c|c|}
\hline SNPs & Cases (\%) & Controls (\%) & $p$-value & OR $(95 \% \mathrm{Cl})$ & padj & OR $(95 \% \mathrm{Cl}) \mathrm{adj}^{\dagger}$ \\
\hline \multicolumn{7}{|l|}{ rs4787050: } \\
\hline$-\mathrm{AA}$ & $413(63.8)$ & $473(70.1)$ & & 1.00 (ref) & & 1.00 (ref) \\
\hline$-A G$ & $206(31.8)$ & $189(28.0)$ & 0.067 & $1.248(0.984-1.583)$ & 0.072 & $1.244(0.981-1.578)$ \\
\hline$-G G$ & $28(4.3)$ & $13(1.9)$ & 0.008 & $2.467(1.261-4.825)$ & 0.008 & $2.471(1.262-4.836)$ \\
\hline $\begin{array}{l}\text { Dominant model } \\
A G+G G \text { vs } A A\end{array}$ & & & 0.016 & $1.327(1.054-1.670)$ & 0.017 & $1.323(1.051-1.665)$ \\
\hline A allele & $1032(79.8)$ & $1135(84.1)$ & & 1.00 (ref) & & \\
\hline G allele & $262(20.2)$ & $215(15.9)$ & 0.004 & $1.340(1.098-1.635)$ & & \\
\hline \multicolumn{7}{|l|}{ rs8045980: } \\
\hline$-\mathrm{CC}$ & $231(35.7)$ & $304(45.0)$ & & 1.00 (ref) & & 1.00 (ref) \\
\hline$-A C$ & $336(52.0)$ & $306(45.3)$ & 0.002 & $1.445(1.147-1.820)$ & 0.002 & $1.448(1.150-1.825)$ \\
\hline C allele & $798(61.7)$ & $914(67.7)$ & & 1.00 (ref) & & \\
\hline A allele & $496(38.3)$ & $436(32.3)$ & 0.001 & $1.303(1.110-1.529)$ & & \\
\hline
\end{tabular}

\section{Table 3. Association of single-nucleotide polymorphisms in RBFOX1 and lung adenocarcinoma risk.}

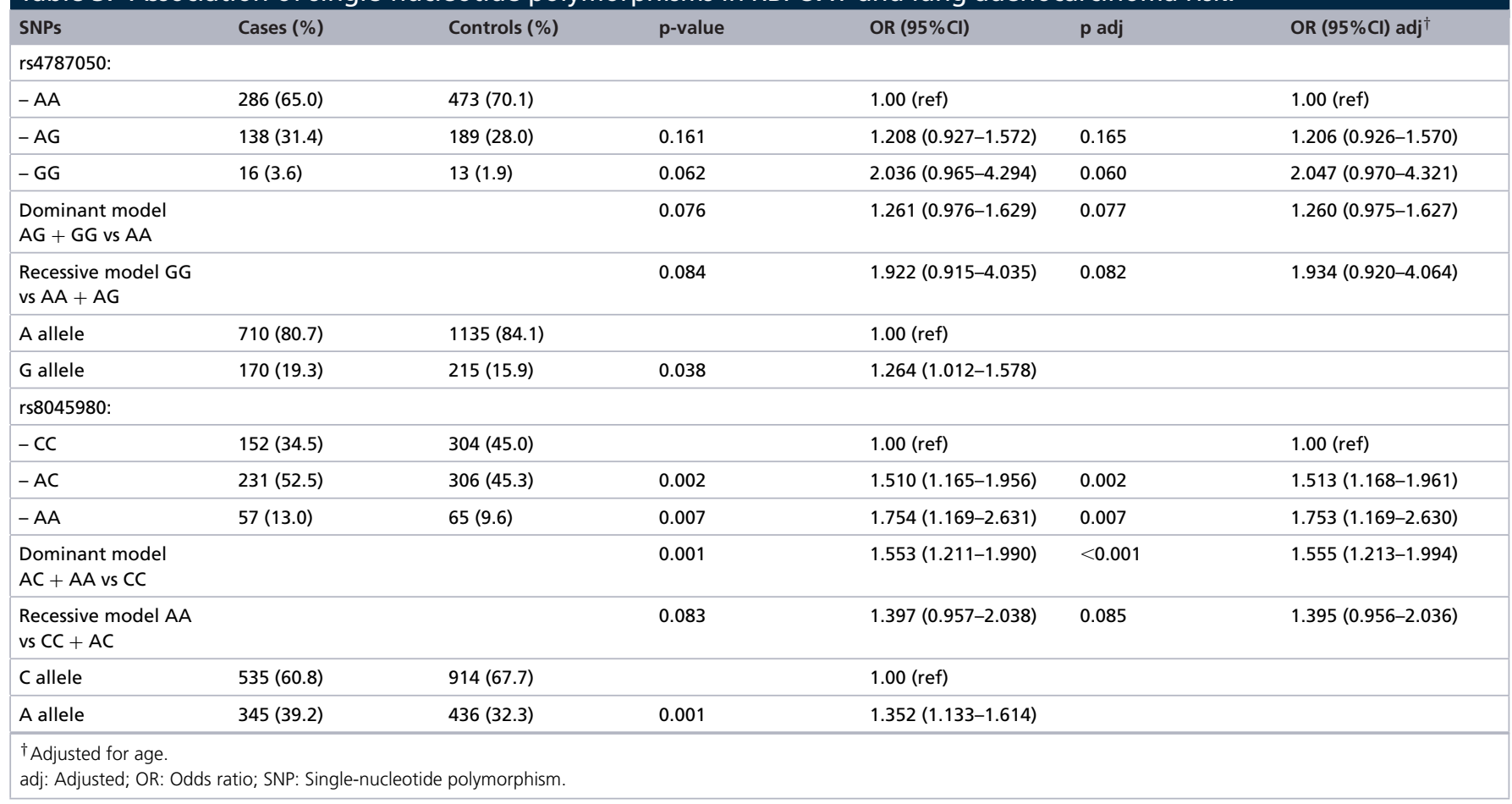

those with the A allele $(\mathrm{OR}=1.264,95 \% \mathrm{CI}=1.012-1.578 ; \mathrm{p}=0.038)$. For rs8045980, the association between SNP and lung adenocarcinoma risk was similar to that between SNP and lung cancer risk. AC genotype, AA genotype and the dominant model $(\mathrm{AC}+\mathrm{AA})$ were related to a significantly increased risk of lung adenocarcinoma in contrast to that for homozygous wild CC genotype (adjusted OR $=1.513,95 \%$ CI $=1.168-1.961 ; \mathrm{p}=0.002$; 
Table 4. The association of single-nucleotide polymorphisms in RBFOX1 and the susceptibility of small cell lung cancer.

\begin{tabular}{|c|c|c|c|c|c|c|}
\hline SNPS & Cases (\%) & Controls (\%) & $p$-value & OR $(95 \% \mathrm{Cl})$ & $p$ adj & OR $\left(95 \%\right.$ Cl) $\operatorname{adj}^{\dagger}$ \\
\hline \multicolumn{7}{|l|}{ rs4787050: } \\
\hline$-\mathrm{AA}$ & $51(54.3)$ & $473(70.1)$ & & 1.00 (ref) & & 1.00 (ref) \\
\hline$-A G$ & $36(38.3)$ & $189(28.0)$ & 0.015 & $1.767(1.117-2.795)$ & 0.015 & $1.770(1.119-2.802)$ \\
\hline$-G G$ & $7(7.4)$ & $13(1.9)$ & 0.001 & $4.994(1.906-13.084)$ & 0.001 & $5.016(1.913-13.149)$ \\
\hline $\begin{array}{l}\text { Dominant model } \\
A G+G G \text { vs } A A\end{array}$ & & & 0.002 & $1.974(1.274-3.059)$ & 0.002 & $1.978(1.276-3.067)$ \\
\hline A allele & $138(73.4)$ & $1135(84.1)$ & & 1.00 (ref) & & \\
\hline G allele & $50(26.6)$ & $215(15.9)$ & $<0.001$ & $1.913(1.341-2.727)$ & & \\
\hline \multicolumn{7}{|l|}{ rs8045980: } \\
\hline$-\mathrm{CC}$ & $38(40.4)$ & $304(45.0)$ & & 1.00 (ref) & & $1.00($ ref) \\
\hline$-A C$ & $43(45.7)$ & $306(45.3)$ & 0.621 & $1.124(0.707-1.789)$ & 0.625 & $1.123(0.706-1.787)$ \\
\hline C allele & $119(63.3)$ & $914(67.7)$ & & 1.00 (ref) & & \\
\hline A allele & $69(36.7)$ & $436(32.3)$ & 0.228 & $1.216(0.885-1.670)$ & & \\
\hline
\end{tabular}

adjusted $\mathrm{OR}=1.753,95 \% \mathrm{CI}=1.169-2.630 ; \mathrm{p}=0.007$; adjusted $\mathrm{OR}=1.555,95 \% \mathrm{CI}=1.213-1.994 ; \mathrm{p}<0.001$, respectively). Moreover, rs8045980-A allele showed a significant increase in the risk of lung adenocarcinoma compared with the $\mathrm{C}$ allele $(\mathrm{OR}=1.352,95 \% \mathrm{CI}=1.133-1.614 ; \mathrm{p}=0.001)$.

In squamous cell carcinoma, we did not find any association between the two SNPs and lung cancer risk (Supplementary Table 1). In small cell lung cancer, all models of rs 4787050 were associated with lung cancer risk (Table 4). Using the AA genotype as a reference, small cell lung cancer susceptibility was found to be significantly higher in subjects with AG genotype, GG genotype and the dominant model (AG + GG; adjusted OR = 1.770, $95 \% \mathrm{CI}=1.119-2.802 ; \mathrm{p}=0.015 ;$ adjusted $\mathrm{OR}=5.016,95 \% \mathrm{CI}=1.913-13.149 ; \mathrm{p}=0.001 ;$ adjusted $\mathrm{OR}=1.978$, $95 \% \mathrm{CI}=1.276-3.067 ; \mathrm{p}=0.002$, respectively). In the recessive model, the GG genotype of rs 4787050 showed a significant increase in the risk of small cell lung cancer compared with the reference (AA + AG; adjusted OR = 4.109, $95 \% \mathrm{CI}=1.595-10.582 ; \mathrm{p}=0.003)$. Individuals carrying the $\mathrm{G}$ allele were more likely to develop small cell lung cancer than those carrying the A allele $(\mathrm{OR}=1.913,95 \% \mathrm{CI}=1.341-2.727$; $\mathrm{p}<0.001)$. However, we did not observe any significant association between rs 8045980 polymorphism and small cell lung cancer susceptibility. A linkage disequilibrium (LD) analysis was conducted between rs4787050 and rs8045980. The LD plots, as shown in Figure 1, indicated that there exists no strong LD between rs4787050 and rs8045980.

\section{SNPs in RBFOX1 \& exposure to cooking oil fume, as well as their interaction on lung cancer susceptibility}

Table 1 presented a significant difference between the case and control groups in exposure to cooking oil fume $(\mathrm{p}=0.002)$. Further unconditional logistic regression analysis demonstrated that exposure to cooking oil fume was associated with an increased risk of lung cancer $(\mathrm{OR}=1.833,95 \% \mathrm{CI}=1.261-2.665 ; \mathrm{p}=0.002)$. On analyzing the correlation between different histological types of lung cancer and exposure to cooking oil fume, we found a more significant correlation between lung adenocarcinoma and cooking oil fume exposure than that between lung squamous cell carcinoma and small cell lung cancer (lung adenocarcinoma: $\mathrm{OR}=1.756,95 \% \mathrm{CI}=1.173-2.630$; $\mathrm{p}=0.006$; lung squamous cell carcinoma: $\mathrm{OR}=2.002,95 \% \mathrm{CI}=1.032-3.960 ; \mathrm{p}=0.040$; small cell lung cancer: $\mathrm{OR}=2.126,95 \% \mathrm{CI}=0.939-4.813 ; \mathrm{p}=0.070)$.

Table 5 detailed the interaction between SNPs and exposure to cooking oil fume on lung cancer risk. For rs4787050 and rs8045980, the results of the crossover analysis were similar (adjusted OR $=3.292,95 \% \mathrm{CI}=1.743-$ 
(A)

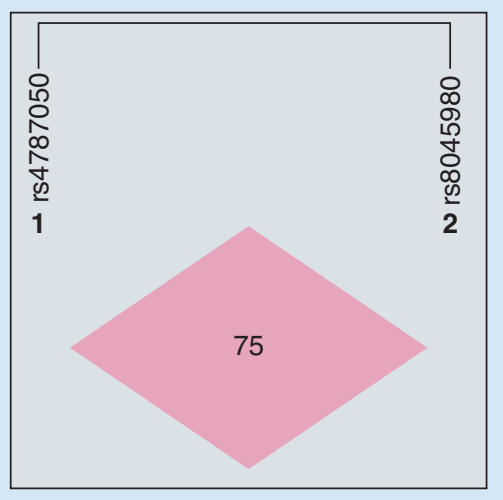

(B)

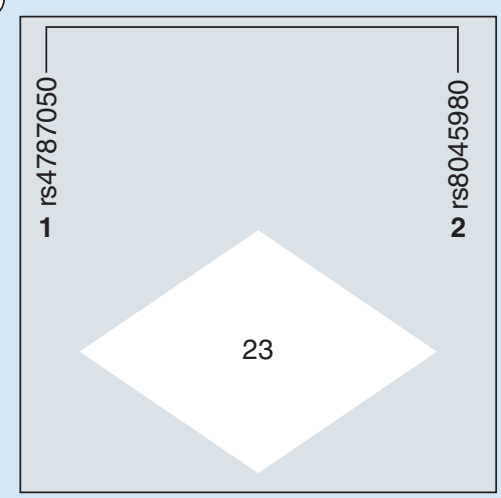

Figure 1. The linkage disequilibrium analysis. (A) $D^{\prime}$ linkage map of single-nucleotide polymorphisms in $R B F O X 1$; (B) $\mathrm{R}^{2}$ linkage map of SNPs in RBFOX1.

Table 5. Interaction between single-nucleotide polymorphisms and cooking oil fume exposure on lung cancer

\section{susceptibility.}

\begin{tabular}{|c|c|c|c|c|c|c|c|}
\hline SNPs & $\begin{array}{l}\text { Environmental } \\
\text { exposure }\end{array}$ & Cases & Controls & p-value & OR $(95 \% \mathrm{Cl})$ & $p$ adj & OR $(95 \% \mathrm{Cl}) \mathrm{adj}^{\dagger}$ \\
\hline \multicolumn{8}{|c|}{ rs4787050 } \\
\hline AA & Non-exposure & 107 & 141 & & 1.00 (ref) & & 1.00 (ref) \\
\hline AG/GG & Non-exposure & 60 & 60 & 0.216 & $\begin{array}{l}1.318 \\
(0.851-2.040)\end{array}$ & 0.187 & $1.344(0.866-2.084)$ \\
\hline AA & Exposure & 60 & 49 & 0.039 & $\begin{array}{l}1.614 \\
(1.025-2.539)\end{array}$ & 0.042 & $1.601(1.016-2.523)$ \\
\hline AG/GG & Exposure & 39 & 16 & $<0.001$ & $\begin{array}{l}3.212 \\
(1.704-6.054)\end{array}$ & $<0.001$ & $3.292(1.743-6.218)$ \\
\hline \multicolumn{8}{|c|}{ rs8045980 } \\
\hline $\mathrm{CC}$ & Non-exposure & 54 & 92 & & 1.00 (ref) & & 1.00 (ref) \\
\hline $\mathrm{AC} / \mathrm{AA}$ & Non-exposure & 113 & 109 & 0.009 & $\begin{array}{l}1.766 \\
(1.153-2.706)\end{array}$ & 0.008 & $1.789(1.166-2.745)$ \\
\hline CC & Exposure & 31 & 36 & 0.200 & $\begin{array}{l}1.467 \\
(0.816-2.636)\end{array}$ & 0.213 & $1.453(0.807-2.614)$ \\
\hline $\mathrm{AC} / \mathrm{AA}$ & Exposure & 68 & 29 & $<0.001$ & $\begin{array}{l}3.995 \\
(2.306-6.920)\end{array}$ & $<0.001$ & $4.053(2.336-7.033)$ \\
\hline
\end{tabular}

$\dagger^{\dagger}$ Adjusted for age.

Adj: Adjusted; OR:Odds ratio; SNP: Single-nucleotide polymorphism.

6.218; $\mathrm{p}<0.001$; adjusted $\mathrm{OR}=4.053,95 \% \mathrm{CI}=2.336-7.033 ; \mathrm{p}<0.001$, respectively). The results of the crossover analysis suggested gene-environment interaction might exist, so we carried out further analyses according to the additive scale and multiplicative scale (Table $6 \&$ Supplementary Table 2, respectively). However, the results of analyses based on the additive scale and the multiplicative scale did not demonstrate any statistical significance.

\section{Discussion}

In recent years, many studies have demonstrated that SNPs and gene mutations play a critical role in the occurrence and development of tumors [19-21]. RBFOX1 encodes a splicing regulator, which affects splicing efficiency of target alternative exons, many in transcripts for other splicing regulators by binding intronic UGCAUG elements [30,31]. Therefore, RBFOX1 mutations might increase the risk of many diseases [32-34]. Tada et al. found that the loss of heterozygosity in $R B F O X 1$ is an independent prognostic factor in gastric cancer, especially during the early stage, through high-density SNP array [12]. Several recent studies have shown that RBFOX1 is associated with various cancers such as breast cancer, colorectal cancer, malignant mesothelioma, non-small-cell lung cancer, choroid 
Table 6. Interaction between single-nucleotide polymorphisms in RBFOX1 and cooking oil fume exposure on lung cancer susceptibility, analyzed based on additive scale.

\begin{tabular}{|c|c|c|c|}
\hline Measure & Estimate & Lower & Upper \\
\hline \multicolumn{4}{|c|}{ rs4787050 } \\
\hline RERI & 1.281 & -0.742 & 3.303 \\
\hline AP & 0.399 & -0.033 & 0.830 \\
\hline$S$ & 2.375 & 0.676 & 8.345 \\
\hline \multicolumn{4}{|c|}{ rs8045980 } \\
\hline RERI & 1.762 & -0.186 & 3.709 \\
\hline AP & 0.441 & 0.108 & 0.774 \\
\hline$S$ & 2.428 & 0.897 & 6.574 \\
\hline
\end{tabular}

plexus tumors, gliomagenesis, liver cancer - among others [10-18]. Several intronic SNPs in RBFOX1 have also been explored. For instance, rs6500843 was related to survival in breast cancer patients [11], whereas rs12446308 was associated with survival in early-stage-non-small cell lung cancer [14]. On the other hand, rs8051518 was related to a markedly increased risk of somatic mutation in SF3B1 via functional alterations in RNA splicing, which might be why RBFOX1 is involved in tumorigenesis [19]. Finally, Rajaram and colleagues demonstrated that RBFOXI is a gene with increased genomic instability (common fragile sites) [35], but to date no association has been studied between RBFOX1 SNPs with lung cancer susceptibility.

We selected rs 4787050 and rs 8045980 located in the intron region of $R B F O X 1$ for the following reasons: first, according to the dbSNP database [35], minor allele frequencies of rs4787050 and rs8045980 in RBFOX1 were 0.163 and 0.329 , respectively, in the Chinese population, which were both $>0.05$. The minor allele frequencies of the two SNPs were consistent with the data in our controls ( 0.159 for rs4787050 and 0.323 for rs8045980). Second, based on the prediction of RegulomeDB [36] and ensemble [37] databases, rs4787050 and rs8045980 may affect transcription factor binding and splicing efficiency. Therefore, the two SNPs may create a difference in the gene function and may be related to diseases. Third, no LD exists between rs4787050 and rs8045980 according to the ensemble database [37], which was consistent with the results of the LD analysis in the current study. Fourth, 4787050 and rs8045980 are expression quantitative trait locus of RBFOX1, according to the rSNPBase database [38]. The eQTL was closely related to gene function and diseases [39]. Finally, according to the results of our previous genome-wide association study, an association between the two SNPs (4787050 and rs8045980) and lung cancer susceptibility may exist. These SNPs were included in a GWAS chip, however, they were not mentioned in published GWAS studies of lung cancer.

We used the statistical software (Quanto) to calculate the sample size for this study based on preliminary experimental results (the first 200 people with lung cancer and 200 people without lung cancer). The sample size we estimated were 1248 and 882 for rs 4787050 and rs 8045980 , respectively. The case control ratio in a case-control study should be less than or equal to one. In this case the control sample size is greater than or equal to the case sample size. Therefore, in the present study, we assessed the association between the two SNPs rs 4787050 and rs 8045980 and lung cancer risk in 647 patients and 675 control subjects, all of whom were female nonsmokers and belonged to genetically unrelated Han population in northeast China. The vast majority of the people with lung cancer studied were adenocarcinomas. Our results suggested the association of the SNPs rs4787050 and rs8045980 in RBFOX1 with significantly increased lung cancer susceptibility. For rs4787050, individuals with AG/GG genotype were 1.32-times more likely to develop lung cancer than those with AA genotype. For rs8045980, subjects with AC/AA genotype were 1.48-times more likely to develop lung cancer than those with CC genotype. Further stratification analyses based on histological types were performed to demonstrate that rs4787050 and rs8045980 in RBFOX1 remain associated with a significantly elevated lung cancer risk in the adenocarcinoma subgroup. In small cell lung cancer, the presence of rs 4787050 polymorphism might elevate the risk of lung cancer, whereas in squamous cell carcinoma, the two SNPs did not show statistical associations with lung cancer. However, because a relatively limited sample population was recruited in the small cell lung cancer and squamous cell carcinoma subgroups, the results warrant further confirmatory studies. The results of LD analysis indicated no strong LD between rs4787050 and rs8045980. The gene-environment interactions could play a pivotal role in the pathogenesis of complex diseases [40-42]. Previous studies [6,43] have suggested that cooking oil fume could inflict DNA damage and 
affect the development of lung cancer. Furthermore, our results demonstrated that cooking oil fume exposure was associated with an increased risk of lung cancer. Therefore, we further analyzed the interaction between the two SNPs and cooking oil fume exposure on the susceptibility to lung cancer. However, our results did not demonstrate any statistically significant interaction. Because the sample size was relatively small for the analysis of the involved environmental factors, the interaction between the 2 SNPs and cooking oil fume exposure warrants further studies in larger cohorts.

In the present study, we attempted to make the study as reliable as possible. First, the genotypic frequencies of rs4787050 and rs8045980 in control subjects conformed to the Hardy-Weinberg equilibrium, indicating the good representativeness of research objects. Second, we performed statistical analyses after adjusting for age. Third, power analysis rates were 83.34 and $91.22 \%$ for both rs 4787050 and rs8045980, respectively (as calculated by Quanto software). Fourth, all case patients had primary lung cancer that was treated without chemotherapy, and all patients were newly diagnosed, which could successfully avoid the Neyman bias. In addition, Berkson bias (admission bias) was effectively avoided by enrolling the case patients and control subjects from the same hospital. However, there are some limitations to our study. First, selection and recall biases may exist because of the hospital-based, case-control study design. Moreover, regional population-based association studies are important for revealing population-specific SNPs and disease risk. For example, Palmer et al. [44] found PLCE1 rs2274223 polymorphism is not associated with gastric cancer risk in the Caucasian population, but Zhang et al. [45] revealed it was associated with gastric cancer risk in the Asian population. However, our conclusions apply only to the northeast Han-Chinese population, thereby necessitating further research in other areas or populations in the future.

\section{Conclusion}

Our study revealed that the two SNPs rs4787050 and rs8045980 were associated with a significantly increased risk of lung cancer and also that exposure to cooking oil fume could elevate the susceptibility to lung cancer among northeast Chinese female nonsmokers. However, no significant interactions were observed between the two SNPs and exposure to cooking oil fume on lung cancer risk.

\section{Summary points}

- Northeast Chinese female nonsmokers were selected as subjects, including 647 people with lung cancer and 675 people without lung cancer.

- We studied the relationship between single-nucleotide polymorphisms (SNPs; rs4787050 and rs8045980) in RBFOX1 and lung cancer risk, as well as studied interaction between the two SNPs and cooking oil fume exposure on lung cancer risk in northeast Chinese female nonsmokers.

- Results of the current study indicated that the two SNPs (rs4787050 and rs8045980) were associated with a significantly increased risk of lung cancer.

- We found that cooking oil fume exposure could elevate the susceptibility of lung cancer in northeast Chinese female nonsmokers. However, no significant interactions were observed between the two SNPs and cooking oil fume exposure on lung cancer risk.

- The current study demonstrated first that rs4787050 and rs8045980 in RBFOX1 might be meaningful as the novel biomarker for lung cancer susceptibility.

\section{Acknowledgments \\ We are very grateful to all people for their participation.}

\section{Financial \& competing interests disclosure}

This study is supported by grant no.81502878 from National Natural Science Foundation of China, no.201501017 from the Doctoral Scientific Research Foundation of Liaoning Province and no. 2015020721 from the Natural Science Foundation of Liaoning Province in China. The authors have no other relevant affiliations or financial involvement with any organization or entity with a financial interest in or financial conflict with the subject matter or materials discussed in the manuscript apart from those disclosed.

No writing assistance was utilized in the production of this manuscript.

Ethical conduct of research

This research was supported by the Institutional Review Board of China Medical University, and informed consent was obtained from all participants. 
Open access

This work is licensed under the Attribution-NonCommercial-NoDerivatives 4.0 Unported License. To view a copy of this license, visit http://creativecommons.org/licenses/by-nc-nd/4.0/

\section{References}

Papers of special note have been highlighted as: $\bullet$ of interest; $\bullet \bullet$ of considerable interest

1. Siegel RL, Miller KD, Jemal A. Cancer statistics, 2017. CA Cancer J. Clin. 67(1), 7-30 (2017).

-. The study summarized and predicted the cancer development situation.

2. Siegel RL, Miller KD, Jemal A. Cancer statistics, 2016. CA Cancer J. Clin. 66(1), 7-30 (2016).

3. Torre LA, Bray F, Siegel RL, Ferlay J, Lortet-Tieulent J, Jemal A. Global cancer statistics, 2012. CA Cancer J. Clin. 65(2), 87-108 (2015).

4. Chen W, Zheng R, Baade PD et al. Cancer statistics in China, 2015. CA Cancer J. Clin. 66(2), 115-132 (2016).

5. Jha P. Avoidable global cancer deaths and total deaths from smoking. Nat. Rev. Cancer 9(9), 655-664 (2009).

6. Couraud S, Zalcman G, Milleron B, Morin F, Souquet PJ. Lung cancer in never smokers - a review. Eur. J. Cancer 48(9), 1299-1311 (2012).

7. Granville CA, Dennis PA. An overview of lung cancer genomics and proteomics. Am. J. Respir. Cell Mol. Biol. 32(3), 169-176 (2005).

8. Kuroyanagi H. Fox-1 family of RNA-binding proteins. Cell. Mol. Life Sci. 66(24), 3895-3907 (2009).

9. Jin Y, Suzuki H, Maegawa $S$ et al. A vertebrate RNA-binding protein Fox-1 regulates tissue-specific splicing via the pentanucleotide GCAUG. Embo J. 22(4), 905-912 (2003).

10. Klorin G, Rozenblum E, Glebov O et al. Integrated high-resolution array CGH and SKY analysis of homozygous deletions and other genomic alterations present in malignant mesothelioma cell lines. Cancer Genet. 206(5), 191-205 (2013).

11. Fagerholm R, Schmidt MK, Khan S et al. The SNP rs6500843 in 16p13.3 is associated with survival specifically among chemotherapy-treated breast cancer patients. Oncotarget 6(10), 7390-7407 (2015).

-• Indicates that rs6500843 in RBFOX1 was related to survival in breast cancer patients.

12. Tada M, Kanai F, Tanaka Y et al. Prognostic significance of genetic alterations detected by high-density single nucleotide polymorphism array in gastric cancer. Cancer Sci. 101(5), 1261-1269 (2010).

13. Andersen CL, Lamy P, Thorsen $\mathrm{K}$ et al. Frequent genomic loss at chr16p13.2 is associated with poor prognosis in colorectal cancer. Int J. Cancer 129(8), 1848-1858 (2011).

14. Huang YT, Heist RS, Chirieac LR et al. Genome-wide analysis of survival in early-stage non-small-cell lung cancer. J. Clin. Oncol. 27(16), 2660-2667 (2009).

- Demonstrates rs12446308 in RBFOX1 was associated with survival in early-stage non-small-cell lung cancer.

15. Mampaey E, Fieuw A, Van Laethem T et al. Focus on 16p13.3 locus in colon cancer. PLoS ONE 10(7), e0131421 (2015).

16. Japp AS, Gessi M, Messing-Junger $M$ et al. High-resolution genomic analysis does not qualify atypical plexus papilloma as a separate entity among choroid plexus tumors. J. Neuropathol. Exp. Neurol. 74(2), 110-120 (2015).

17. Hu J, Ho AL, Yuan L et al. From the cover: neutralization of terminal differentiation in gliomagenesis. Proc. Natl Acad. Sci. USA 110(36), 14520-14527 (2013).

18. Ding D, Lou X, Hua D et al. Recurrent targeted genes of hepatitis B virus in the liver cancer genomes identified by a next-generation sequencing-based approach. PLoS Genet 8(12), e1003065 (2012).

19. Qiu F, Yang L, Lu X et al. The MKK7 p.Glu116Lys rare variant serves as a predictor for lung cancer risk and prognosis in Chinese. PLoS Genet. 12(3), e1005955 (2016).

20. Chen D, Zhong F, Chen Y. Association of calcium/calmodulin-dependent protein kinase kinase1 rs 7214723 polymorphism with lung cancer risk in a Chinese population. Biosci. Rep. 37(4), BSR20170762 (2017).

-• Shows that cooking oil fume exposure might increase lung cancer risk in Chinese female nonsmokers.

21. Singh A, Singh N, Behera D, Sharma S. Polymorphism in XRCC1 gene modulates survival and clinical outcomes of advanced north Indian lung cancer patients treated with platinum-based doublet chemotherapy. Med. Oncol. 34(4), 64 (2017).

- The study found gene-environment interaction might play a vital role in lung cancer risk.

22. Carter H, Marty R, Hofree M et al. Interaction landscape of inherited polymorphisms with somatic events in cancer. Cancer Discov. 7(4), 410-423 (2017).

23. Xue Y, Jiang Y, Jin S, Li Y. Association between cooking oil fume exposure and lung cancer among Chinese nonsmoking women: a meta-analysis. Onco. Targets Ther. 9, 2987-2992 (2016).

24. Le Marchand L, Wilkens LR. Design considerations for genomic association studies: importance of gene-environment interactions. Cancer Epidemiol. Biomarkers Prev. 17(2), 263-267 (2008). 
25. Yin Z, Cui Z, Ren Y et al. Association between polymorphisms in pre-miRNA genes and risk of lung cancer in a Chinese non-smoking female population. Lung Cancer 94, 15-21 (2016).

26. Ren Y, Yin Z, Li K et al. TGFbeta-1 and TGFBR2 polymorphisms, cooking oil fume exposure and risk of lung adenocarcinoma in Chinese nonsmoking females: a case control study. BMC Med. Genet. 16(1), 22 (2015).

27. Tiret L, Cambien F. Departure from Hardy-Weinberg equilibrium should be systematically tested in studies of association between genetic markers and disease. Circulation 92(11), 3364-3365 (1995).

28. Li Z, Zhang Z, He Z et al. A partition-ligation-combination-subdivision EM algorithm for haplotype inference with multiallelic markers: update of the SHEsis . Cell Res. 19(4), 519-523 (2009).

29. Andersson T, Alfredsson L, Källberg H, Zdravkovic S, Ahlbom A. Calculating measures of biological interaction. Eur. J. Epidemiol. 20(7), 575-579 (2005).

30. Fernandez-Castillo N, Gan G, Van Donkelaar MMJ et al. RBFOX1, encoding a splicing regulator, is a candidate gene for aggressive behavior. Eur. Neuropsychopharmacol. doi:10.1016/j.euroneuro.2017.11.012 (2017).

31. Conboy JG. Developmental regulation of RNA processing by Rbfox proteins. Wiley Interdiscip. Rev RNA 8(2), (2017).

32. Lee JA, Damianov A, Lin CH et al. Cytoplasmic Rbfox1 regulates the expression of synaptic and autism-related genes. Neuron 89(1), 113-128 (2016).

33. Gao C, Ren S, Lee JH et al. RBFox1-mediated RNA splicing regulates cardiac hypertrophy and heart failure. J. Clin. Invest. 126(1), 195-206 (2016).

34. He KY, Wang H, Cade BE. Rare variants in fox-1 homolog A (RBFOXI) are associated with lower blood pressure. PLoS Genet. 13(3), e1006678 (2017).

35. Sherry ST, Ward M, Sirotkin K. dbSNP-database for single nucleotide polymorphisms and other classes of minor genetic variation. Genome Res 9(8), 677-679 (1999).

- Demonstrates that RBFOX1 is a gene with increased genomic instability (common fragile sites).

36. Boyle AP, Hong EL, Hariharan M et al. Annotation of functional variation in personal genomes using RegulomeDB. Genome Res. 22(9), 1790-1797 (2012).

37. Cunningham F, Achuthan P, Akanni W et al. Ensembl 2019. Nucleic Acids Res. 47(D7), D745-D751 (2018).

38. Guo L, Wang J. rSNPBase 3.0: an updated database of SNP-related regulatory elements, element-gene pairs and SNP-based gene regulatory networks. Nucleic Acids Res. 46(D1), D1111-D1116 (2018).

39. Fagny M, Paulson JN, Kuijjer ML et al. Exploring regulation in tissues with eQTL networks. Proc. Natl Acad. Sci. USA 114(37), E7841-E7850 (2017).

40. Song N, Shin A. Effects of interactions between common genetic variants and smoking on colorectal cancer. BMC Cancer 17(1), 869 (2017).

41. Baldwin DA, Sarnowski CP, Reddy SA et al. Development of a genotyping microarray for studying the role of gene-environment interactions in risk for lung cancer. J. Biomol. Tech. 24(4), 198-217 (2013).

42. Huang J, Liu Y, Vitale S et al. On meta- and mega-analyses for gene-environment interactions. Genet. Epidemiol. 41(8), 876-886 (2017).

43. Tokiwa H, Sera N, Nakashima A, Nakashima K, Nakanishi Y, Shigematu N. Mutagenic and carcinogenic significance and the possible induction of lung cancer by nitro aromatic hydrocarbons in particulate pollutants. Environ. Health Perspect. 102(Suppl. 4), 107-110 (1994).

44. Palmer AJ, Lochhead P, Hold GL et al. Genetic variation in C20orf54, PLCE1 and MUC1 and the risk of upper gastrointestinal cancers in Caucasian populations. Eur. J. Cancer Prev. 21(6), 541-544 (2012).

45. Zhang H, Jin G, Li H et al. Genetic variants at 1q22 and 10q23 reproducibly associated with gastric cancer susceptibility in a Chinese population. Carcinogenesis 32(6), 848-852 (2011). 